\title{
ON THE SOLVABILITY OF A SYSTEM OF WAVE AND BEAM EQUATIONS
}

\section{JUHA BERKOVITS}

Received 27 October 2000

We prove new existence results for linearly coupled system of wave and beam equations. The main concept is the matrix spectrum which is a natural extension of standard definition. Using invariant subspaces together with degree theoretic argument we obtain information about the range of the abstract operator.

\section{Introduction}

We consider a system of wave and beam operators with linear coupling and damping having the form

$$
\begin{gathered}
\frac{\partial^{2} u}{\partial t^{2}}-\frac{\partial^{2} u}{\partial x^{2}}-a_{11} u-a_{12} v-g_{1}(t, x, u, v)=h_{1}(t, x) \quad \text { in } \Omega, \\
\frac{\partial^{2} v}{\partial t^{2}}+\frac{\partial^{4} v}{\partial x^{4}}+\beta \frac{\partial v}{\partial t}-a_{21} u-a_{22} v-g_{2}(t, x, u, v)=h_{2}(t, x) \quad \text { in } \Omega, \\
u(t, 0)=u(t, \pi)=0, \quad t \in] 0,2 \pi[, \\
\left.v(t, 0)=v(t, \pi)=v_{x x}(t, 0)=v_{x x}(t, \pi)=0, \quad t \in\right] 0,2 \pi[, \\
u(\cdot, x), v(\cdot, x) \quad \text { are } 2 \pi \text {-periodic in } t
\end{gathered}
$$

where $h=\left(h_{1}, h_{2}\right)$ is a given function in $L_{2}\left(\Omega ; \mathbb{R}^{2}\right)$ with $\left.\Omega=\right] 0,2 \pi[\times] 0, \pi[$, $\beta>0$, the function $g(t, x, s)=\left(g_{1}(t, x, s), g_{2}(t, x, s)\right)$ from $\Omega \times \mathbb{R}^{2}$ to $\mathbb{R}^{2}$ is $2 \pi$ periodic in $t$, measurable in $(t, x)$ for each $s \in \mathbb{R}^{2}$ and continuous in $s$ for almost all $(t, x) \in \Omega$. Moreover, we assume that $g(t, x, \cdot)$ has at most linear growth. With suitable nonlinearity $g(1.1)$ provides a reasonable model for a suspension bridge, where the main cable is described as a vibrating string and the road bed as a vibrating beam. 
We will study the existence of weak solutions and therefore it is relevant to transform system (1.1) into the operator equation

$$
\mathscr{L} u-\mathscr{A} u-\mathcal{N}(u)=h, \quad u \in D(\mathscr{L})
$$

in $\mathscr{H}=\left[L_{2}(\Omega)\right]^{2}$, where $\mathcal{N}$ is the Nemytskii operator generated by $g, \mathscr{A}$ is the constant multiplication operator induced by the coupling matrix $A:=\left(a_{l k}\right)$ and $\mathscr{L}: D(\mathscr{L}) \subset \mathscr{H} \rightarrow \mathscr{H}$ is the abstract realization of the linear differential operator. We define the matrix spectrum of $\mathscr{L}$ as the set

$$
\sigma_{M}(\mathscr{L})=\left\{A \in \mathbb{R}_{2 \times 2} \mid(\mathscr{L}-\mathscr{A})^{-1}: \mathscr{H} \longrightarrow \mathscr{H} \nexists \text { or is not bounded }\right\}
$$

The set $\sigma_{M}(\mathscr{L})$ is closed in $\mathbb{R}_{2 \times 2}$ and hence the "resolving set" of $\mathscr{L}$ is divided into open components. We will apply the extension of the Leray-Schauder degree introduced by Berkovits and Mustonen [4] for a class of mappings related to our model problem. If $A \notin \sigma_{M}(\mathscr{L})$, we can use the homotopy argument to obtain nonresonance results for (1.2) (see [8]). In this note we deal with the resonance case $A \in \sigma_{M}(\mathscr{L})$. Using suitable reduction to invariant subspaces we can find solution for (1.2), provided $\mathcal{N}$ and $h$ satisfy some auxiliary symmetry conditions. Indeed, if the coupled wave-beam operator $\mathscr{L}-\mathscr{A}$ is completely reduced by a closed linear subspace $V$ and $N(V) \subset V$, any solution of the reduced equation

$$
\left.\mathscr{L}\right|_{V} u-\left.\mathscr{A}\right|_{V} u-\left.\mathcal{N}\right|_{V}(u)=h, \quad u \in D(\mathscr{L}) \cap V, h \in V
$$

is also a weak solution for the original equation (1.2). It is possible that $A \in$ $\sigma_{M}(\mathscr{L})$ but $\left.\mathscr{L}\right|_{V}-\left.\mathscr{A}\right|_{V}$ is injective in the subspace $V$ and hence we can apply the known nonresonance results for the reduced equation (1.4).

The method of invariant subspaces is widely used in the study of ordinary differential equations and for a single wave equation by Coron, Vejvoda, among others. (See $[5,9,11,15]$.)

Problem (1.1) has been studied mainly in the case of gradient type nonlinearities $\mathcal{N}$ with no damping, that is, with $\beta=0$. We refer the reader to the papers of Brezis and Nirenberg [10], Amann [1], Mawhin [13, 14], Fonda and Mawhin [12], where also a survey on the recent results and relevant references can be found. For previous results on the existence of the periodic solutions of the systems of wave equations we also refer to $[2,6,7]$.

\section{Prerequisites}

Let $H$ be a real separable Hilbert space with inner product $\langle\cdot, \cdot\rangle$ and corresponding norm $\|\cdot\|$. We recall some basic definitions. A mapping $F: H \rightarrow H$ is

- bounded, if it takes any bounded set into a bounded set,

- demicontinuous, if $u_{j} \rightarrow u$ (norm convergence) implies $F\left(u_{j}\right) \rightarrow F(u)$ (weak convergence), 
- monotone, if $\langle F(u)-F(v), u-v\rangle \geq 0$ for all $u, v \in H$,

- strongly monotone, if there exists $\alpha>0$ such that $\langle F(u)-F(v)$, $u-v\rangle \geq \alpha\|u-v\|^{2}$ for all $u, v \in H$,

- of class $\left(S_{+}\right)$, if for any sequence with $u_{j} \rightarrow u, \lim \sup \left\langle F\left(u_{j}\right)\right.$, $\left.u_{j}-u\right\rangle \leq 0$, it follows that $u_{j} \rightarrow u$,

- quasimonotone, if for any sequence $u_{j} \rightarrow u, \lim \sup \left\langle F\left(u_{j}\right), u_{j}-u\right\rangle \geq 0$.

The class of mappings considered in this paper is given in the following definitions.

Definition 2.1. A linear densely defined operator $L: D(L) \subset H \rightarrow H$ is admissible in $H$, if it is closed, normal, $\operatorname{Im} L=(\operatorname{Ker} L)^{\perp}$ and the inverse $K$ of the restriction of $L$ to $\operatorname{Im} L \cap D(L)$ is compact on $\operatorname{Im} L$.

Denote by $P$ and $Q=I-P$ the orthogonal projections to $\operatorname{Ker} L$ and $\operatorname{Im} L=$ $(\operatorname{Ker} L)^{\perp}$, respectively.

Definition 2.2. A bounded, demicontinuous map $N: H \rightarrow H$ is an admissible perturbation in $H$, if there exists a bounded demicontinuous map $S: H \rightarrow H$ of class $\left(S_{+}\right)$such that $P N=P S$.

We are interested in the case where $L$ is not selfadjoint and therefore we include the complex spectrum of $L$ into consideration. We recall that the complexification $H_{C}=H+i H$ of $H$ has the usual linear structure and inner product $\langle\cdot, \cdot\rangle_{\mathbb{C}}$ induced by $H$. For each $w=u+i v \in H_{\mathbb{C}}$ it is natural to denote $\bar{w}=u-i v$. We define the complex linear operator $L_{\mathbb{C}}: D\left(L_{\mathbb{C}}\right) \subset H_{\mathbb{C}} \rightarrow H_{\mathbb{C}}$ by setting $D\left(L_{\mathbb{C}}\right)=D(L)+i D(L)$ and $L_{\mathbb{C}}(u+i v)=L u+i L v$ for all $u+i v \in$ $D\left(L_{\mathbb{C}}\right)$. It is clear that $\operatorname{Im} L_{\mathbb{C}}=\left(\operatorname{Ker} L_{\mathbb{C}}\right)^{\perp}, L_{\mathbb{C}}$ is normal and its partial inverse $K_{\mathbb{C}}$ is compact. Consequently, $L_{\mathbb{C}}$ has a pure point spectrum $\sigma_{\mathbb{C}}(L)=\left\{\mu_{j}\right\}_{j \in \mathbb{Z}}$ with the corresponding orthonormal basis $\left\{\phi_{j}\right\}_{j \in \mathbb{Z}}$ of $H_{\mathbb{C}}$ such that

$$
L_{\mathbb{C}} \phi_{j}=\mu_{j} \phi_{j} \quad \forall j \in \mathbb{Z} .
$$

Note that for any complex eigenvalue $\mu_{j}=\alpha_{j}+i \beta_{j}$ also its complex conjugate $\bar{\mu}_{j}=\alpha_{j}-i \beta_{j}$ is an eigenvalue with corresponding eigenvector $\bar{\phi}_{j}$. For each $u \in D(L)$ we have the spectral representation

$$
L u=\sum_{j \in \mathbb{Z}} \mu_{j}\left\langle u, \phi_{j}\right\rangle_{\mathbb{C}} \phi_{j} .
$$

For any map $N: H \rightarrow H$ the equation

$$
L u-N(u)=0, \quad u \in D(L),
$$

can be written equivalently as

$$
Q(u-K Q N(u))+P N(u)=0, \quad u \in H .
$$


The equivalence of (2.3) and (2.4) is due to the fact that $K Q-P$ is the right inverse of $L-P$. If $N$ is bounded, demicontinuous and of class $\left(S_{+}\right)$, then there exists a topological degree for mappings of the form $F=Q(I+C)+P N$, where $C$ is compact (see [4]). In fact, it is sufficient that $N$ is admissible, that is, there exists an auxiliary bounded demicontinuous map $S: H \rightarrow H$ of class $\left(S_{+}\right)$such that $P N=P S$. This observation is quite obvious, since only the $P$-component of $N$ appears in $F$. However, it has some interesting implications as we will see later on (see also [3]). The degree theory given in [4] is a unique extension of the classical Leray-Schauder degree. It is single-valued and has the usual properties of degree, such as additivity and invariance under homotopies. Let the corresponding degree function be $d_{H}$. In order to simplify our notations we define a further degree function " $\operatorname{deg}_{H}$ " by setting

$$
\operatorname{deg}_{H}(L-N, G, 0) \equiv d_{H}(Q(I-K Q N)+P N, G, 0)
$$

for any open set $G \subset H$ such that $0 \notin(L-N)(\partial G \cap D(L))$. By the term reference map we refer to any linear injection $L-N_{0}$ with $L$ and $N_{0}$ admissible. For a reference map we have (see [4])

$$
\operatorname{deg}_{H}\left(L-N_{0}, G, y\right) \neq 0 \quad \text { for any } y \in\left(L-N_{0}\right)(D(L) \cap G) \text {. }
$$

\section{On systems}

Let $H$ be a real separable Hilbert space and denote $\mathscr{H}=H^{n}$ with $n \geq 2$. We assume that $L_{k}: D\left(L_{k}\right) \subset H \rightarrow H$ is a linear densely defined closed, normal operator with $\operatorname{Im} L_{k}=\left(\operatorname{Ker} L_{k}\right)^{\perp}$ for each $k=1,2, \ldots, n$. The inverse $K_{k}$ of the restriction of each $L_{k}$ to $\operatorname{Im} L_{k} \cap D\left(L_{k}\right)$ is a bounded linear operator on $\operatorname{Im} L_{k}$. We shall further assume that the inverse $K_{k}$ of each $L_{k}$ is compact and hence each $L_{k}$ is admissible. We define the diagonal operator $\mathscr{L}: D(\mathscr{L}) \subset \mathscr{H} \rightarrow \mathscr{H}$ by setting

$$
\mathscr{L} u=\left(L_{1} u_{1}, L_{2} u_{2}, \ldots, L_{n} u_{n}\right)^{T}, \quad u=\left(u_{1}, u_{2}, \ldots, u_{n}\right)^{T} \in D(\mathscr{L}),
$$

where $D(\mathscr{L})=D\left(L_{1}\right) \times D\left(L_{2}\right) \times \cdots \times D\left(L_{n}\right)$. Now the complexification $\mathscr{H}_{\mathbb{C}}=$ $\mathscr{H}+i \mathscr{H}=\left(H_{\mathbb{C}}\right)^{n}$ and $\mathscr{L}$ as well as $\mathscr{L}_{\mathbb{C}}$ inherits the properties of the component operators. We shall use the notations $\langle\cdot, \cdot\rangle$ and $\|\cdot\|$ for the inner product and norm in any real Hilbert space and the subscript " $\mathbb{C}$ " whenever the norm, inner product or spectrum is complex. For simplicity we shall frequently use the same symbol for an operator and its complexification. The inverse $\mathscr{K}=\mathscr{L}^{-1}: \operatorname{Im} \mathscr{L} \rightarrow$ $\operatorname{Im} \mathscr{L}$ is compact with $\mathscr{K} u=\left(K_{1} u_{1}, \ldots, K_{n} u_{n}\right)^{T}$ for all $u=\left(u_{1}, u_{2}, \ldots, u_{n}\right)^{T} \in$ $\operatorname{Im} \mathscr{L}$. We denote by $\mathscr{P}$ and $\mathscr{Q}$ the orthogonal projections onto $\operatorname{Ker} \mathscr{L}$ and $\operatorname{Im} \mathscr{L}$, respectively. Let $\mathcal{N}: \mathscr{H} \rightarrow \mathscr{H}$ be a (possibly nonlinear) bounded demicontinuous map. As described in Section 2, a topological degree is available for maps of the form $\mathscr{L}-\mathcal{N}$, where $\mathscr{L}$ is admissible and $\mathcal{N}$ is any admissible perturbation. 
Consider first linear maps of the following type. Let $A=\left(a_{l k}\right)$ be a real $n \times n$-matrix and $\mathscr{A}: \mathscr{H} \rightarrow \mathscr{H}$ the constant multiplication operator induced by $A$, that is, for any $u=\left(u_{1}, u_{2}, \ldots, u_{n}\right)^{T} \in \mathscr{H}$

$$
\mathscr{A} u=w=\left(w_{1}, w_{2}, \ldots, w_{n}\right)^{T}
$$

with $w_{l}=\sum_{k=1}^{n} a_{l k} u_{k}, l=1,2, \ldots, n$. Clearly $\sigma(\mathscr{A})=\sigma(A)$, a real point spectrum which may be empty if $n$ is even. Similarly $\sigma_{\mathbb{C}}(\mathscr{A})=\sigma_{\mathbb{C}}(A)$ for the complex spectra. If the matrix $A$ is strictly positive, it is not hard to prove that

$$
\langle\mathscr{A} u, u\rangle \geq \alpha\|u\|^{2} \quad \forall u \in \mathscr{H},
$$

where $\alpha=\min \left\{(A x, x)_{\mathbb{R}^{n}} ;|x|_{\mathbb{R}^{n}}=1\right\}$ is positive. Hence the operator $\mathscr{A}$ is of class $\left(S_{+}\right)$. In order to tackle more specific situations we assume that $\operatorname{dim} \operatorname{Ker} L_{k}$ $=\infty$ for $k=1,2, \ldots, p$ and $\operatorname{dim} \operatorname{Ker} L_{k}<\infty$ for $k=p+1, \ldots, n$, where $0 \leq p \leq n$. If $p=n$ we assume that $A>0$ and if $p=0$, no positivity is needed. For the general case, we formulate the condition:

(PC) The matrix $\left(a_{l k}\right)_{l, k=1}^{p}$ is strictly positive.

We have the following useful result adopted from [3].

Lemma 3.1. Assume that $1 \leq p \leq n$ and the positivity condition $(P C)$ holds. Then there exists a bounded linear operator $S_{A}: \mathscr{H} \rightarrow \mathscr{H}$ of class $\left(S_{+}\right)$such that $\mathscr{P} S_{A}=\mathscr{P} \mathscr{A}$, that is, $\mathscr{A}$ is an admissible linear perturbation.

It is important to realize the meaning of $S_{A}$; it is only needed to guarantee the existence of the topological degree. All concrete calculations will be done with $\mathcal{A}$, not with $S_{A}$. We shall impose a further "common eigenbasis"-assumption:

(CE) The operators $L_{k}, k=1,2, \ldots, n$, have a common complex eigenbasis $\left\{\psi_{j}\right\}_{j \in \Lambda}$.

Here we can assume that the index set $\Lambda \subset \mathbb{Z}$. We denote the corresponding complex eigenvalues by $\left\{\mu_{j}^{(k)}\right\}_{j \in \Lambda}$. Hence $L_{k} \psi_{j}=\mu_{j}^{(k)} \psi_{j}$ for all $j \in \Lambda, k=$ $1,2, \ldots, n$. Although assumption $(\mathrm{CE})$ is very restrictive from the general point of view it can be verified in many applications. It trivially holds in case $L_{1}=$ $L_{2}=\cdots=L_{n}$. For any $u=\left(u_{1}, u_{2}, \ldots, u_{n}\right)^{T} \in \mathscr{H}$ we denote $\vec{u}_{j}=$ $\sum_{k=1}^{n}\left\langle u_{k}, \psi_{j}\right\rangle_{\mathbb{C}} e_{k}$, where $\left\{e_{k}\right\}$ is the standard basis of $\mathbb{R}^{n}$. Hence we can write

$$
u=\sum_{j \in \Lambda} \vec{u}_{j} \psi_{j}, \quad\|u\|^{2}=\sum_{j \in \Lambda}\left\|\vec{u}_{j}\right\|_{\mathbb{C}^{n}}^{2} .
$$

It is easy to see that for any $u \in D(\mathscr{L})$ we have a quasidiagonal representation

$$
(\mathscr{L}-\mathscr{A})(u)=\sum_{j \in \Lambda}\left[\left(M_{j}-A\right) \vec{u}_{j}\right] \psi_{j}
$$

where $M_{j}=\operatorname{diag}\left(\mu_{j}^{(1)}, \mu_{j}^{(2)}, \ldots, \mu_{j}^{(n)}\right)$. By (3.5) we get the following result (cf. [8]). 
Lemma 3.2. Assume (CE). The operator $\mathscr{L}-\mathscr{A}$ is injective if and only if $\operatorname{det}\left(M_{j}-A\right) \neq 0$ for all $j \in \Lambda$. Moreover, an injective operator $\mathscr{L}-\mathscr{A}$ is onto if and only if $\sup _{j}\left\|\left(M_{j}-A\right)^{-1}\right\|<\infty$.

Note that in case $L_{k}=L$ for all $k=1,2, \ldots, n$, the injectivity condition in Lemma 3.2 can be written as $\sigma_{\mathbb{C}}(L) \cap \sigma_{\mathbb{C}}(A)=\emptyset$. By condition (PC) we get the following result (cf. [8]).

LeMma 3.3. Assume (PC), that is, the matrix $\left(a_{l k}\right)_{l, k=1}^{p}$ is strictly positive. If the operator $\mathscr{L}-\mathscr{A}$ is injective, then it is bijective.

We get a simple formula for the norm of $(\mathscr{L}-\mathscr{A})^{-1}$.

Lemma 3.4. Assume that $(C E)$ and $(P C)$ hold. Then $\operatorname{det}\left(M_{j}-A\right) \neq 0$ for all $j \in \mathbb{Z}$, if and only if the operator $\mathscr{L}-\mathscr{A}$ is bijective. Moreover, $(\mathscr{L}-\mathscr{A})^{-1}$ is bounded and

$$
\left\|(\mathscr{L}-\mathscr{A})^{-1}\right\|=\sup _{j}\left\|\left(M_{j}-A\right)^{-1}\right\|<\infty .
$$

In general, the operator $\mathscr{L}-\mathscr{A}$ can be injective without being surjective (cf. [3]). However, in the special case $L_{1}=L_{2}=\cdots=L_{n}$, injectivity implies bijectivity without any positivity condition (see [7]). We recall now the concept of the matrix spectrum introduced in [8]. Given a linear operator $\mathcal{T}$ with domain and range in $\mathscr{H}=H^{n}$, we define the matrix resolvent of the operator $\mathcal{T}$ as the set

$$
\rho_{M}(\mathscr{T})=\left\{A \in \mathbb{R}_{n \times n} \mid \overline{\operatorname{Im}(\mathscr{T}-\mathscr{A})}=\mathscr{H}, \mathscr{T}-\mathscr{A} \text { has a bounded inverse }\right\}
$$

The matrix spectrum of the operator $\mathscr{T}$ is then the set

$$
\sigma_{M}(\mathscr{T})=\left\{A \in \mathbb{R}_{n \times n} \mid A \notin \rho_{M}(\mathscr{T})\right\}
$$

It is not hard to prove that the matrix spectrum $\sigma_{M}(\mathscr{T})$ is a closed set in $\mathbb{R}_{n \times n}$ (see [8]). In the particular case where $\mathscr{L}$ has the properties given at the beginning of the section, that is, $\mathscr{L}$ is an admissible diagonal operator, it is not hard to prove that $\operatorname{Im}(\mathscr{L}-\mathscr{A})$ is closed whenever the positivity condition (PC) holds. Then also the degree theory is available and any invertible operator $\mathscr{L}-\mathscr{A}$ is a reference map. Recall that a linear operator $\mathscr{T}$ is completely reduced by a closed linear subspace $V \subset \mathscr{H}$ if

$$
P_{V}(D(\mathscr{T})) \subset D(\mathscr{T}), \quad \mathscr{T} P_{V} u=P_{V} \mathscr{T} u \quad \forall u \in D(\mathscr{T}),
$$

where $P_{V}$ is the orthogonal projection from $\mathscr{H}$ onto $V$. The use of degree theory in an invariant subspace is justified by the following result. 
LEMMA 3.5. Assume that $L$ and $N$ are admissible in $H, L$ is completely reduced by a closed linear subspace $V \subset H$ and $N(V) \subset V$. Then $\left.L\right|_{V}$ and $\left.N\right|_{V}$ are admissible in $V$.

Proof. Denote $\tilde{L}=\left.L\right|_{V}$ and $\tilde{N}=\left.N\right|_{V}$. It is easy to verify that $\tilde{L}: D(L) \cap V \rightarrow$ $V$ is admissible in $V$ and $\tilde{P}=P P_{V}=P_{V} P$ is the orthogonal projection onto $\operatorname{Ker} \tilde{L}$. Since $N$ is admissible, there exists a bounded demicontinuous map $S$ : $H \rightarrow H$ of class $\left(S_{+}\right)$with $P S=P N$. Denote $\tilde{S}=\left.P_{V} S\right|_{V}$. Then $\tilde{S}: V \rightarrow V$ is a bounded demicontinuous map of class $(S+)$. Moreover, $\tilde{P} \tilde{S}(u)=P_{V} P S(u)=$ $P_{V} P N(u)=\tilde{P} \tilde{N}(u)$ for all $u \in V$. Hence $\tilde{N}$ is admissible in $V$.

In the sequel we will need a generalization of the well-known result $\sigma(\mathcal{T})=$ $\sigma\left(\left.\mathscr{T}\right|_{V}\right) \cup \sigma\left(\left.\mathscr{T}\right|_{V^{\perp}}\right)$. The concept of the matrix spectrum is defined in the space $H^{n}$ and hence it is too narrow to deal with invariant subspaces. Therefore we define more general "spectrum-like" sets. Indeed, let $H$ be any real separable Hilbert space and $T$ a linear operator with domain and range in $H$. Denote

$$
\begin{gathered}
\rho_{\mathrm{BL}}(T)=\{A \in L(H) \mid \overline{\operatorname{Im}(T-A)}=H, T-A \text { has a bounded inverse }\} \\
\sigma_{\mathrm{BL}}(T)=\left\{A \in L(H) \mid A \notin \rho_{\mathrm{BL}}(T)\right\} .
\end{gathered}
$$

Here "BL" stands for "bounded linear" and $L(H)$ is the space of bounded linear operators in $\mathrm{H}$. The set $\sigma_{\mathrm{BL}}(T)$ is closed in $L(H)$. We have in analogy with Lemma 3.3 the following result.

Lemma 3.6. Assume that $L: D(L) \subset H \rightarrow H$ is admissible and $S \in L(H)$ is some admissible linear perturbation. Then $S \in \sigma_{\mathrm{BL}}(L)$ if and only if $L-S$ is not injective.

Clearly $A \in \sigma_{M}(\mathscr{L})$ if and only if $\mathscr{A} \in \sigma_{\mathrm{BL}}(\mathscr{L})$. Moreover, if $\mathscr{L}$ is completely reduced by a closed subspace $V \subset \mathscr{H}$, then the sets $\sigma_{\mathrm{BL}}\left(\left.\mathscr{L}\right|_{V}\right)$ and $\sigma_{\mathrm{BL}}\left(\left.\mathscr{L}\right|_{V^{\perp}}\right)$ are well defined. We obtain the following result.

Lemma 3.7. Assume that $\mathscr{L}$ and $\mathscr{A}$ are admissible and $\mathscr{A}$ is a constant multiplication operator induced by the matrix $A \in \mathbb{R}_{n \times n}$. Assume that both $\mathscr{L}$ and $\mathscr{A}$ are completely reduced by a closed subspace $V \subset H^{n}$. Then

$$
A \in \sigma_{M}(\mathscr{L}) \quad \text { iff }\left.\quad \mathscr{A}\right|_{V} \in \sigma_{\mathrm{BL}}\left(\left.\mathscr{L}\right|_{V}\right) \quad \text { or }\left.\quad \mathscr{A}\right|_{V^{\perp}} \in \sigma_{\mathrm{BL}}\left(\left.\mathscr{L}\right|_{V^{\perp}}\right) \text {. }
$$

The straightforward proof of Lemma 3.7 is omitted here. If the maps $\mathscr{L}$ and $\mathscr{A}$ are admissible, then it is easy to prove that the "geometric multiplicity" of any "eigenmatrix" $A \in \sigma_{M}(\mathscr{L})$ is finite, that is, $\operatorname{dim} \operatorname{Ker}(\mathscr{L}-\mathscr{A})<\infty$. Assuming (CE), that is, the existence of a common complex eigenbasis, we can write $\sigma_{M}(\mathscr{L})=\left(\cup_{j \in \Lambda} \sigma_{j}\right) \cup \sigma_{\infty}$, where $\sigma_{j}=\left\{A \in \mathbb{R}_{n \times n} \mid \operatorname{det}\left(M_{j}-A\right)=0\right\}, j \in \Lambda$, 
222 On the solvability of a system of wave and beam equations

and $\sigma_{\infty}=\left\{A \in \mathbb{R}_{n \times n} \mid A \notin \cup_{j \in \Lambda} \sigma_{j}, \sup _{j}\left\|\left(M_{j}-A\right)^{-1}\right\|=\infty\right\}$. Now $\cup_{j \in \Lambda} \sigma_{j}$ corresponds to the usual point spectrum and $\sigma_{\infty}$ to the continuous spectrum. As noted above, Lemma 3.3 implies $A \notin \sigma_{\infty}$ whenever the positivity condition (PC) holds for $A$ and $\mathscr{L}$ is admissible. By homotopy argument, we get the following basic existence result. Using the results in $[7,8]$ together with Lemmas 3.5 and 3.7 we obtain the following result.

TheOrem 3.8. Assume that $\mathscr{L}$ is admissible, the condition (PC) holds for $A \in$ $\sigma_{M}(\mathscr{L})$ and the operators $\mathscr{L}$ and $\mathscr{A}$ are completely reduced by a closed linear subspace $V \subset \mathcal{H}$. Assume that $\mathcal{N}: \mathscr{H} \rightarrow \mathcal{H}$ is such that $\mathcal{N}(V) \subset V$ and $(\mathscr{A}+$ $\mathcal{N})\left.\right|_{V}$ is admissible in $V$. If $\left.\mathscr{A}\right|_{V} \notin \sigma_{\mathrm{BL}}\left(\left.\mathscr{L}\right|_{V}\right)$ and

$$
\limsup _{u \in V,\|u\| \rightarrow \infty} \frac{\|\mathcal{N}(u)\|}{\|u\|}<\frac{1}{\left\|\left(\left.(\mathscr{L}-\mathscr{A})\right|_{V}\right)^{-1}\right\|},
$$

then the equation

$$
\mathscr{L} u-\mathscr{A} u-\mathcal{N}(u)=h, \quad u \in D(\mathscr{L}) \cap V
$$

admits at least one solution for any $h \in V$.

\section{Wave-beam system}

We consider first a linear system of wave and beam equations with linear coupling and damping having the form

$$
\begin{gathered}
\frac{\partial^{2} u}{\partial t^{2}}-\frac{\partial^{2} u}{\partial x^{2}}-a_{11} u-a_{12} v=h_{1}(t, x) \quad \text { in } \Omega, \\
\frac{\partial^{2} v}{\partial t^{2}}+\frac{\partial^{4} v}{\partial x^{4}}+\beta \frac{\partial v}{\partial t}-a_{21} u-a_{22} v=h_{2}(t, x) \quad \text { in } \Omega, \\
u(t, 0)=u(t, \pi)=0, \quad t \in] 0,2 \pi[, \\
\left.v(t, 0)=v(t, \pi)=v_{x x}(t, 0)=v_{x x}(t, \pi)=0, \quad t \in\right] 0,2 \pi[, \\
u(\cdot, x), v(\cdot, x) \quad \text { are } 2 \pi \text {-periodic in } t,
\end{gathered}
$$

where $h=\left(h_{1}, h_{2}\right)$ is a given function in $L_{2}\left(\Omega ; \mathbb{R}^{2}\right)$ with $\left.\Omega=\right] 0,2 \pi[\times] 0, \pi[$ and $\beta>0$. Here, the coupling matrix is

$$
A=\left(\begin{array}{ll}
a_{11} & a_{12} \\
a_{21} & a_{22}
\end{array}\right) .
$$

Denoting $H=L_{2}(\Omega)$ and $\phi_{j k}(t, x)=(1 / \pi) \sin (j x) \exp (i k t),(t, x) \in \Omega, j \in$ $\mathbb{Z}_{+}, k \in \mathbb{Z}$, the set $\left\{\phi_{j k}\right\}$ forms an orthonormal basis in $H_{\mathbb{C}}$. The wave operator $\partial_{t t}-\partial_{x x}$ with periodic Dirichlet boundary conditions has in $L_{2}(\Omega)$ the abstract realization

$$
L_{1} u=\sum_{j, k} \lambda_{j k}^{(1)}\left\langle u, \phi_{j k}\right\rangle_{\mathbb{C}} \phi_{j k}
$$


with $\lambda_{j k}^{(1)}=j^{2}-k^{2}, j \in \mathbb{Z}_{+}, k \in \mathbb{Z}$ and

$$
D\left(L_{1}\right)=\left\{u \in L_{2}(\Omega)\left|\sum_{j, k}\right| j^{2}-\left.k^{2}\right|^{2}\left|\left\langle u, \phi_{j k}\right\rangle_{\mathbb{C}}\right|^{2}<\infty\right\} .
$$

Clearly $L_{1}$ is selfadjoint, $\operatorname{Ker} L_{1}$ is infinite-dimensional and $L_{1}$ has a pure point spectrum $\sigma\left(L_{1}\right)=\left\{\lambda_{j k}^{(1)} \mid j \in \mathbb{Z}_{+}, k \in \mathbb{Z}\right\}$. Note that the spectrum is unbounded from below and from above. The beam operator (with damping) $\partial_{t t}+\partial_{x x x x}+\beta \partial_{t}$ has an analogous realization

$$
L_{2} v=\sum_{j, k}\left(\lambda_{j k}^{(2)}+i \beta k\right)\left\langle v, \phi_{j k}\right\rangle_{\mathbb{C}} \phi_{j k}, \quad v \in D\left(L_{2}\right),
$$

where $\lambda_{j k}^{(2)}=j^{4}-k^{2}, j \in \mathbb{Z}_{+}, k \in \mathbb{Z}$. If $\beta>0$, then the operator $L_{2}$ is normal, $\operatorname{Ker} L_{2}=\{0\}$ and $\sigma_{\mathbb{C}}\left(L_{2}\right)=\left\{j^{4}-k^{2}+i \beta k \mid j \in \mathbb{Z}_{+}, k \in \mathbb{Z}\right\}$. In case $\beta=0$ the operator $L_{2}$ is selfadjoint with infinite-dimensional kernel. We will always assume that $\beta>0$. The diagonal operator $\mathscr{L}=\operatorname{diag}\left(L_{1}, L_{2}\right)$ is defined on $D(\mathscr{L})=D\left(L_{1}\right) \times D\left(L_{2}\right) \subset \mathscr{H}=H^{2}$. Then $\mathscr{L}$ is normal with compact (partial) inverse from $\operatorname{Im} \mathscr{L}$ into $\operatorname{Im} \mathscr{L}$. A vector $w=(u, v)^{T} \in\left[L_{2}(\Omega)\right]^{2}$ is a weak solution of the wave-beam system (4.1) if and only if it is a solution of the operator equation

$$
\mathscr{L} w-\mathscr{A} w=h, \quad w=(u, v)^{T} \in D(\mathscr{L}) \subset \mathscr{H}=H^{2},
$$

where $h=\left(h_{1}, h_{2}\right)^{T} \in \mathscr{H}$. Denote $M_{j k}=\operatorname{diag}\left(\lambda_{j k}^{(1)}, \lambda_{j k}^{(2)}+i \beta k\right)$. The operator $\mathscr{L}-\mathscr{A}$ is injective if and only if

$$
\operatorname{det}\left(M_{j k}-A\right)=\operatorname{det}\left(\begin{array}{cc}
\lambda_{j k}^{(1)}-a_{11} & -a_{12} \\
-a_{21} & \lambda_{j k}^{(2)}+i \beta k-a_{22}
\end{array}\right) \neq 0
$$

for all $j \in \mathbb{Z}_{+}, k \in \mathbb{Z}$. Moreover, assuming the positivity condition (PC), which in this case means that $a_{11}>0$, the operator $\mathscr{A}$ is admissible and by Lemma 3.3 the injectivity of $\mathscr{L}-\mathscr{A}$ implies its surjectivity and hence $\mathscr{L}-\mathscr{A}$ is a reference map. We now study more closely the case $A \in \sigma_{M}(\mathscr{L})$. If $a_{11}>0$, then $\operatorname{dim} \operatorname{Ker}(\mathscr{L}-\mathscr{A})<\infty$. In fact, for a wave-beam system (with $\beta>0$ ) it is easy to see that $\operatorname{dim} \operatorname{Ker}(\mathscr{L}-\mathscr{A})=\infty$ if and only if $a_{11}=0$ and $a_{12} a_{21}=0$. Note that the condition $\operatorname{det}\left(M_{j 0}-A\right)=0$ is equivalent to $j^{6}-a_{11} j^{4}-a_{22} j^{2}+\operatorname{det} A=0$. Moreover, $\operatorname{det}\left(M_{j k}-A\right)=0, k \neq 0$, only in the special case, where $a_{11}=\lambda_{j k}^{(1)}$ and $a_{12} a_{21}=0$.

\section{Existence results}

Consider now the linearly coupled system (1.1) of wave and beam equations with some nonlinear perturbation. Indeed, let the given function $g(t, x, s)=$ $\left(g_{1}(t, x, s), g_{2}(t, x, s)\right)$ from $\Omega \times \mathbb{R}^{2}$ to $\mathbb{R}^{2}$ be $2 \pi$-periodic in $t$, measurable in 
224 On the solvability of a system of wave and beam equations

$(t, x)$ for each $s \in \mathbb{R}^{2}$ and continuous in $s$ for a. a. $(t, x) \in \Omega$. Assume that $g$ satisfies the growth condition

$$
|g(t, x, s)| \leq c_{0}|s|+k_{0}(t, x)
$$

for all $s \in \mathbb{R}^{2}$ and a.a. $(t, x) \in \Omega$ with some constant $c_{0} \geq 0$ and $k_{0} \in L_{2}(\Omega)$. We consider a system of a wave equation and a beam equation with linear coupling and damping with $\beta>0$ and $a_{11}>0$. Assume that $A \in \sigma_{M}(\mathscr{L})$. Then $\operatorname{dim} \operatorname{Ker}(\mathscr{L}-\mathscr{A})<\infty$. Denote $\Lambda_{0}=\left\{(j, k) \in \mathbb{Z}_{+} \times \mathbb{Z} \mid \operatorname{det}\left(M_{j k}-A\right)=0\right\}$ and

$$
W_{A}=\operatorname{sp}_{\mathbb{C}}\left\{\psi_{j, k} e_{l} \mid l=1,2,(j, k) \in \Lambda_{0}\right\} \cap \mathscr{H} .
$$

Then $\operatorname{Ker}(\mathscr{L}-\mathscr{A}) \subset W_{A}$ and the operators $\mathscr{L}$ and $\mathscr{A}$ are completely reduced by the finite-dimensional subspace $W_{A}$. In order to apply Theorem 3.8, the main problem is to find natural conditions ensuring the condition " $\mathcal{N}(V) \subset V$ " in Theorem 3.8. We will deal with three separate cases: $g=g(t, s), g=g(x, s)$, and $g=g(s)$.

(A) Assume that $\beta>0, a_{11}>0, \mathscr{A}+\mathcal{N}$ is admissible, $A \in \sigma_{M}(\mathscr{L}), g=$ $g(t, s)$, that is, $g$ is independent of $x$, and $g(t, s)$ is odd in $s$. For any $r \geq 2$, $r \in \mathbb{Z}_{+}$we define the space

$$
\begin{aligned}
V_{r}= & \left\{u \in \mathscr{H} \mid u\left(t, x+\frac{2 \pi}{r}\right)=u(t, x) \text { for a.a. } t \in\right] 0,2 \pi[, x \in] 0, \pi-\frac{2 \pi}{r}[, \\
& \left.u(t, x)=-u\left(t, \frac{2 \pi}{r}-x\right) \text { for a.a } t \in\right] 0,2 \pi[, x \in] 0, \frac{2 \pi}{r}[\} \\
= & \overline{\operatorname{sp}_{\mathbb{C}}}\left\{\psi_{j k} e_{l} \mid \frac{j}{r} \in \mathbb{Z}_{+}, k \in \mathbb{Z}, l=1,2\right\} \cap \mathcal{H} .
\end{aligned}
$$

Now the operators $\mathscr{L}$ and $\mathscr{A}$ are completely reduced by $V_{r}$. Moreover, we can take $r$ such that $\left\{(j, k) \mid j / r \in \mathbb{Z}_{+}, k \in \mathbb{Z}\right\} \cap \Lambda_{0}=\emptyset$ (for instance $r>\max \{j \mid$ $\left.\left.(j, k) \in \Lambda_{0}\right\}\right)$. Then $\left.\mathscr{A}\right|_{V_{r}} \notin \sigma_{\mathrm{BL}}\left(\left.\mathscr{L}\right|_{V_{r}}\right)$. It is easy to see that $N\left(V_{r}\right) \subset V_{r}$ and hence it is relevant to consider the reduced equation

$$
\left.\mathscr{L}\right|_{V_{r}} u-\left.\mathscr{A}\right|_{V_{r}} u-\left.N\right|_{V_{r}}(u)=h, \quad u \in D(L) \cap V_{r},
$$

for any $h \in V_{r}$. Any solution of (5.4) is also a weak solution for the original equation. The reduced operator $\left.\mathscr{L}\right|_{V_{r}}-\left.\mathscr{A}\right|_{V_{r}}$ is injective and hence we can apply Theorem 3.8. Indeed, we obtain the following result.

Theorem 5.1. Assume that $\beta>0, a_{11}>0, A \in \sigma_{M}(\mathscr{L})$. Moreover, assume that $g=g(t, s), g(t, \cdot)$ is odd and $\mathscr{A}+\mathcal{N}$ is admissible. If $\left\{(j, k) \mid j / r \in \mathbb{Z}_{+}, k \in\right.$ $\mathbb{Z}\} \cap \Lambda_{0}=\emptyset$ and there exist constants $\eta_{r} \geq 0$ and $C_{r} \geq 0$ such that

$$
\eta_{r}<\left\|\left(\left.(\mathscr{L}-\mathscr{A})\right|_{V_{r}}\right)^{-1}\right\|^{-1}, \quad\left\|\left.\mathcal{N}\right|_{V_{r}}(u)\right\| \leq \eta_{r}\|u\|+C_{r} \quad \forall u \in V_{r},
$$


then the equation

$$
\mathscr{L} u-\mathscr{A} u-\mathcal{N}(u)=h, \quad u \in D(L) \cap V_{r},
$$

admits at least one solution for any $h \in V_{r}$.

Proof. By the assumptions of the theorem $\mathcal{N}\left(V_{r}\right) \subset V_{r}$ and

$$
\limsup _{u \in V_{r},\|u\| \rightarrow \infty} \frac{\|\mathcal{N}(u)\|}{\|u\|}<\frac{1}{\left\|\left(\left.(\mathscr{L}-\mathscr{A})\right|_{V_{r}}\right)^{-1}\right\|},
$$

and hence the conclusion follows from Theorem 3.8.

As a direct consequence of the above theorem we get the following result.

COROLlary 5.2. If

$$
c_{0}<\left\|\left(\left.(\mathscr{L}-\mathscr{A})\right|_{V_{r}}\right)^{-1}\right\|^{-1},
$$

where $c_{0} \geq 0$ is the constant in the growth condition (5.1), then Theorem 5.1 holds with $\eta_{r}=c_{0}$.

Note that

$$
\left\|\left(\left.(\mathscr{L}-\mathscr{A})\right|_{V_{r}}\right)^{-1}\right\|=\sup _{j / r \in \mathbb{Z}_{+}, k \in \mathbb{Z}}\left\|\left(M_{j k}-A\right)^{-1}\right\| .
$$

Unfortunately, it seems difficult to find out whether or not the equation admits a solution for any $h \in \operatorname{Ker}(\mathscr{L}-\mathscr{A})^{\perp}$ or even for any $h \in W_{A}^{\perp}$. For a given concrete $A \in \sigma_{M}(\mathscr{L})$ it is easy to find out all the values of $r \in \mathbb{Z}_{+}$for which $\left.\mathscr{A}\right|_{V_{r}} \notin \sigma_{\mathrm{BL}}\left(\left.\mathscr{L}\right|_{V_{r}}\right)$.

(B) Assume now that $\beta>0, a_{11}>0, \mathscr{A}+\mathcal{N}$ is admissible, $A \in \sigma_{M}(\mathscr{L})$ and $g=g(x, s)$, that is, $g$ is independent of $t$. Moreover, assume that $\operatorname{det}\left(M_{j 0}-A\right) \neq$ 0 for all $j \in \mathbb{Z}_{+}$. This means that $j^{6}-a_{11} j^{4}-a_{22} j^{2}+\operatorname{det} A \neq 0$ for all $j \in \mathbb{Z}_{+}$ and hence $k \neq 0$ for all $(j, k) \in \Lambda_{0}$. Since $A \in \sigma_{M}(\mathscr{L})$ we necessarily have $a_{11} \in \sigma\left(L_{1}\right)$ and $a_{12} a_{21}=0$. In this special case we define for any $r \in \mathbb{Z}_{+}$ the space

$$
\begin{aligned}
E_{r} & =\left\{u \in \mathscr{H} \mid u\left(t+\frac{2 \pi}{r}, x\right)=u(t, x) \text { for a.a. } t \in\right] 0,2 \pi-\frac{2 \pi}{r}[, x \in] 0, \pi[\} \\
& =\overline{\operatorname{sp}_{\mathbb{C}}}\left\{\psi_{j k} \mid j \in \mathbb{Z}_{+}, \frac{k}{r} \in \mathbb{Z}\right\} \cap \mathscr{H} .
\end{aligned}
$$

The operators $\mathscr{L}$ and $\mathscr{A}$ are completely reduced by $E_{r}$. Taking $r \in \mathbb{Z}_{+}$such that $\left\{(j, k) \mid j \in \mathbb{Z}_{+}, k / r \in \mathbb{Z}\right\} \cap \Lambda_{0}=\emptyset$ it is clear that $\left.\mathscr{A}\right|_{E_{r}} \notin \sigma_{\mathrm{BL}}\left(\left.\mathscr{L}\right|_{E_{r}}\right)$. Since $g$ is independent of $t$ we have $N\left(E_{r}\right) \subset E_{r}$. Hence we get the following result. 
Theorem 5.3. Assume that $\beta>0, a_{11}>0, A \in \sigma_{M}(\mathscr{L})$. Moreover, assume that $g=g(x, s)$ and $\mathscr{A}+\mathcal{N}$ is admissible. If $\left\{(j, k) \mid j \in \mathbb{Z}_{+}, k / r \in \mathbb{Z}\right\} \cap \Lambda_{0}=\emptyset$ and there exist constants $\eta_{r} \geq 0$ and $C_{r} \geq 0$ such that

$$
\eta_{r}<\left\|\left(\left.(\mathscr{L}-\mathscr{A})\right|_{E_{r}}\right)^{-1}\right\|^{-1}, \quad\left\|\left.\mathcal{N}\right|_{E_{r}}(u)\right\| \leq \eta_{r}\|u\|+C_{r} \quad \forall u \in E_{r},
$$

then the equation

$$
\mathscr{L} u-\mathscr{A} u-\mathcal{N}(u)=h, \quad u \in D(L) \cap E_{r},
$$

admits at least one solution for any $h \in E_{r}$.

Note that in case $\mathcal{N}(0) \neq 0$, Theorem 5.3 gives nontrivial solution for the equation $\mathscr{L} u-\mathscr{A} u-\mathcal{N}(u)=0, u \in D(L)$. However, since always $E_{r} \cap E_{r^{\prime}} \neq\{0\}$ the above result cannot be used to find multiple solutions for equation $\mathscr{L} u-\mathscr{A} u-$ $\mathcal{N}(u)=0$. Of course, an analogous corollary as in case (A) can be formulated.

(C) Assume now that $\beta>0, A \in \sigma_{M}(\mathscr{L})$ is arbitrary (no positivity condition!) and $g=g(s)$. We assume only that $g$ satisfies the Caratheodory conditions and the growth condition (5.1). Define first the subspace

$$
\begin{aligned}
Z_{0} & =\{u \in \mathscr{H} \mid u(t+\pi, \pi-x)=u(t, x) \text { for a.a. } t \in] 0, \pi[, x \in] 0, \pi[\} \\
& =\overline{\operatorname{sp}_{\mathbb{C}}}\left\{\psi_{j k} e_{l} \mid j \in \mathbb{Z}_{+}, k \in \mathbb{Z} \text { with } j+k \text { is odd, } l=1,2\right\} \cap \mathscr{H} .
\end{aligned}
$$

The operators $\mathscr{L}$ and $\mathscr{A}$ are completely reduced by $Z_{0}$ and $\mathcal{N}\left(Z_{0}\right) \subset Z_{0}$. The most remarkable fact is that $\left.\operatorname{dim} \operatorname{Ker} \mathscr{L}\right|_{Z_{0}}=\{0\}$ implying that the inverse of $\left.\mathscr{L}\right|_{Z_{0}}$ is compact. Thus we can employ Leray-Schauder degree in the space $Z_{0}$ and no monotonicity is needed. Moreover, it is easy to see that $\operatorname{dim} \operatorname{Ker}\left(\left.(\mathscr{L}-\mathscr{A})\right|_{Z_{0}}\right)<$

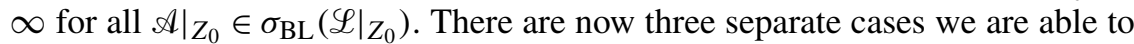
deal with.

(i) First, if $\mathscr{A}_{Z_{0}} \notin \sigma_{\mathrm{BL}}\left(\left.\mathscr{L}\right|_{Z_{0}}\right)$ then we obtain the following variant: if

$$
c_{0}<\left\|\left((\mathscr{L}-\mathscr{A}) \mid z_{0}\right)^{-1}\right\|^{-1},
$$

where $c_{0} \geq 0$ is the constant in the growth condition (5.1), then the equation

$$
\mathscr{L} u-\mathscr{A} u-\mathcal{N}(u)=h, \quad u \in D(L) \cap Z_{0},
$$

admits at least one solution for any $h \in Z_{0}$.

(ii) If $\mathscr{A} \mid Z_{0} \in \sigma_{\mathrm{BL}}\left(\left.\mathscr{L}\right|_{Z_{0}}\right)$ and $g$ is odd we denote $X_{r}=Z_{0} \cap V_{r}$, where $r \in \mathbb{Z}_{+}$is such that $\left\{(j, k) \mid j / r \in \mathbb{Z}_{+}, k \in \mathbb{Z}\right\} \cap \Lambda_{0}=\emptyset$. In analogy with Theorem 5.1 we get the result: if

$$
c_{0}<\left\|\left(\left.(\mathscr{L}-\mathscr{A})\right|_{X_{r}}\right)^{-1}\right\|^{-1},
$$

then the equation

$$
\mathscr{L} u-\mathscr{A} u-\mathcal{N}(u)=h, \quad u \in D(L) \cap X_{r},
$$

admits at least one solution for any $h \in X_{r}$. 
(iii) Assume that $\mathscr{A}_{Z_{0}} \in \sigma_{\mathrm{BL}}\left(\left.\mathscr{L}\right|_{Z_{0}}\right)$, but $\operatorname{det}\left(M_{j 0}-A\right) \neq 0$ for all $j \in \mathbb{Z}_{+}, j$ odd. Hence $k \neq 0$ for all $(j, k) \in \Lambda_{0}, j+k$ odd. Moreover, necessarily $a_{12} a_{21}=$ 0 and $a_{11}=\lambda_{j k}$ for some pair $(j, k) \in \mathbb{Z}_{+} \times \mathbb{Z}$ with $j+k$ odd and $k \neq 0$. Denoting $Y_{r}=Z_{0} \cap E_{r}$, where $\left\{(j, k) \mid j \in \mathbb{Z}_{+}, k / r \in \mathbb{Z}, j+k\right.$ odd $\} \cap \Lambda_{0}=\emptyset$, we get as in case (B): if

$$
c_{0}<\left\|\left(\left.(\mathscr{L}-\mathscr{A})\right|_{Y_{r}}\right)^{-1}\right\|^{-1}
$$

then the equation

$$
\mathscr{L} u-\mathscr{A} u-\mathcal{N}(u)=h, \quad u \in D(L) \cap Y_{r},
$$

admits at least one solution for any $h \in Y_{r}$.

\section{References}

[1] H. Amann, On the unique solvability of semilinear operator equations in Hilbert spaces, J. Math. Pures Appl. (9) 61 (1982), no. 2, 149-175. MR 83k:47049. Zbl 501.47024 .

[2] J. Berkovits, Local bifurcation results for systems of semilinear equations, J. Differential Equations 133 (1997), no. 2, 245-254. MR 98a:47067. Zbl 877.58017.

[3] On the Leray-Schauder formula and bifurcation, J. Differential Equations 173 (2001), 451-469. CMP 1834122 .

[4] J. Berkovits and V. Mustonen, An extension of Leray-Schauder degree and applications to nonlinear wave equations, Differential Integral Equations 3 (1990), no. 5, 945-963. MR 91j:35179. Zbl 724.47024.

[5] - On multiple solutions for a class of semilinear wave equations, Nonlinear Anal. 16 (1991), no. 5, 421-434. MR 92d:35195. Zbl 729.35081.

[6] _ On systems of telegraph equations, Function Spaces, Differential Operators and Nonlinear Analysis (Paseky nad Jizerou, 1995) (J. Rakosnik, ed.), Prometheus, Prague, 1996, pp. 125-140. MR 98k:35126. Zbl 863.35056.

[7] _ On nonresonance for systems of semilinear wave equations, Nonlinear Anal. 29 (1997), no. 6, 627-638. MR 98f:47067. Zbl 887.35013.

[8] J. Berkovits, V. Mustonen, and M. Tienari, Systems of wave equations: matrix spectrum and nonresonance, Function Spaces, Differential Operators and Nonlinear Analysis (Pudasjärvi, 1999) (V. Mustonen and J. Rakosnik, eds.), Acad. Sci. Czech Repub., Prague, 2000, pp. 9-18. CMP 1755 292. Zbl 0965.47049.

[9] H. Brézis, Periodic solutions of nonlinear vibrating strings and duality principles, Bull. Amer. Math. Soc. (N.S.) 8 (1983), no. 3, 409-426. MR 84e:35010. Zbl 515.35060.

[10] H. Brézis and L. Nirenberg, Characterizations of the ranges of some nonlinear operators and applications to boundary value problems, Ann. Scuola Norm. Sup. Pisa Cl. Sci. (4) 5 (1978), no. 2, 225-326. MR 58\#23813. Zbl 386.47035.

[11] J. M. Coron, Periodic solutions of a nonlinear wave equation without assumption of monotonicity, Math. Ann. 262 (1983), no. 2, 273-285. MR 84d:35010. Zbl 489.35061.

[12] A. Fonda and J. Mawhin, Iterative and variational methods for the solvability of some semilinear equations in Hilbert spaces, J. Differential Equations 98 (1992), no. 2, 355-375. MR 93h:47071. Zbl 761.34047. 
228 On the solvability of a system of wave and beam equations

[13] J. Mawhin, Conservative systems of semilinear wave equations with periodicDirichlet boundary conditions, J. Differential Equations 42 (1981), no. 1, 116128. MR 83b:35105. Zbl 475.35063.

[14] Periodic solutions of some semilinear wave equations and systems: a survey, Chaos Solitons Fractals 5 (1995), no. 9, 1651-1669. MR 96m:35224.

[15] O. Vejvoda, Partial Differential Equations, Noordhoff, Sijthoff, 1981.

Juha Berkovits: Department of Mathematical Sciences, University of Oulu, P.O. Box 3000, FIN-90014 Oulu, Finland

E-mail address: juha.berkovits@oulu.fi 


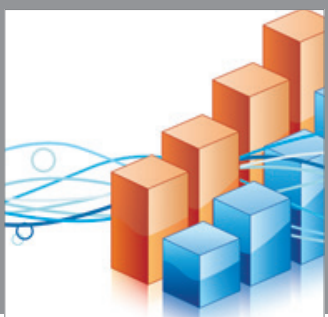

Advances in

Operations Research

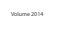

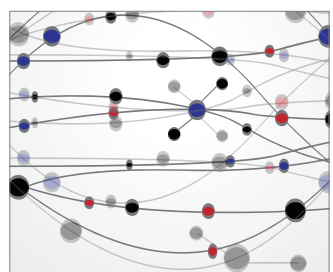

\section{The Scientific} World Journal
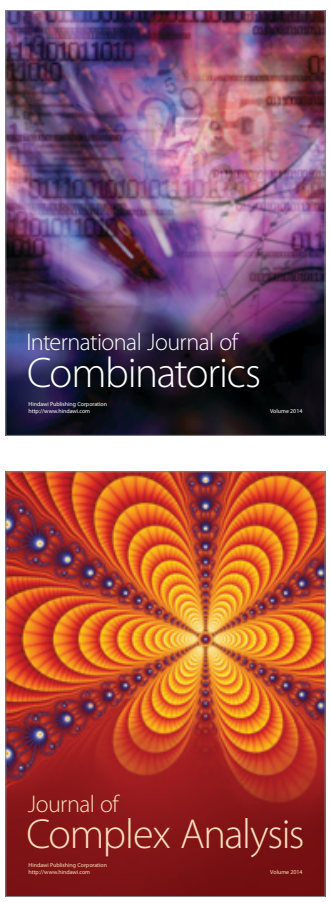

International Journal of

Mathematics and

Mathematical

Sciences
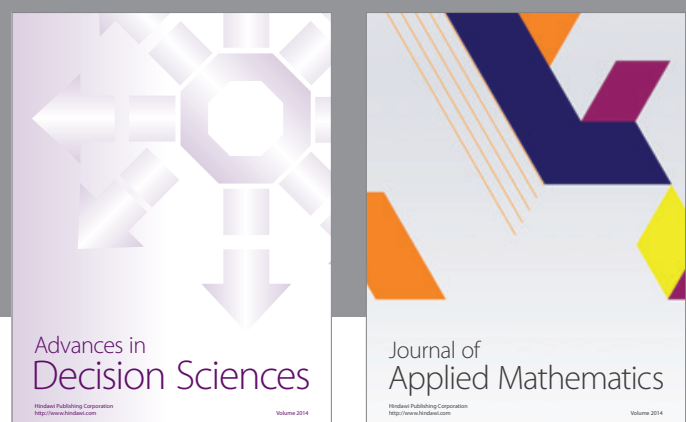

Journal of

Applied Mathematics
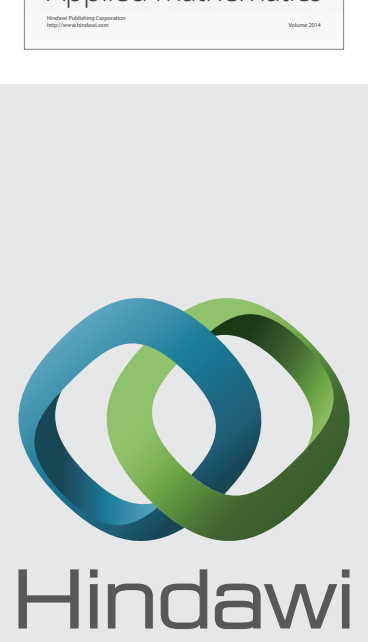

Submit your manuscripts at http://www.hindawi.com
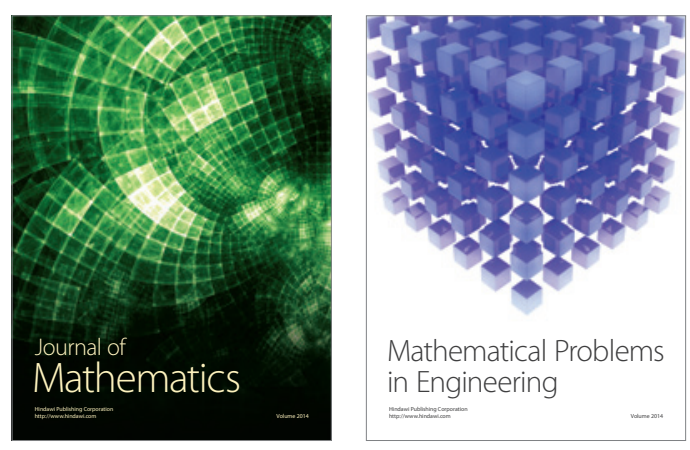

Mathematical Problems in Engineering
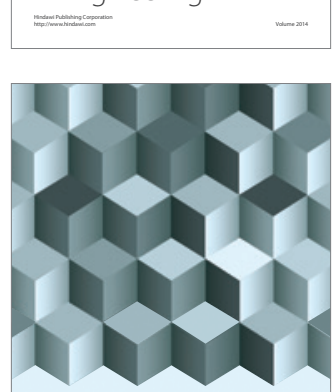

Journal of

Function Spaces
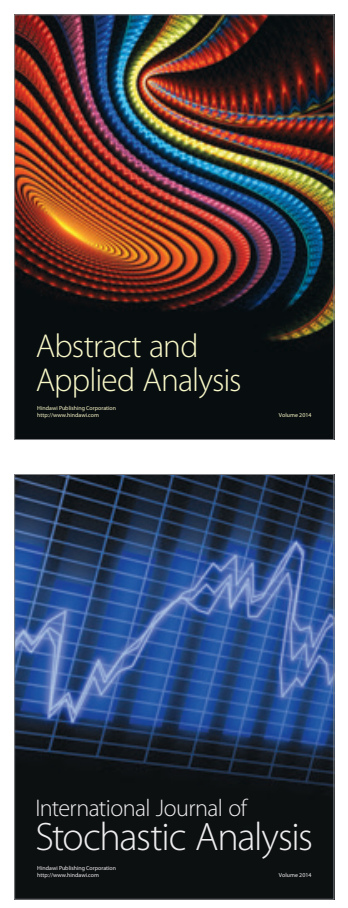

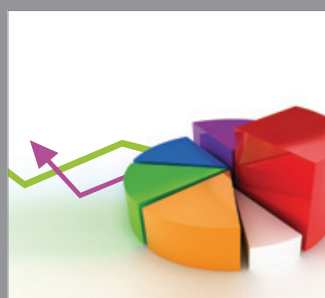

ournal of

Probability and Statistics

Promensencen
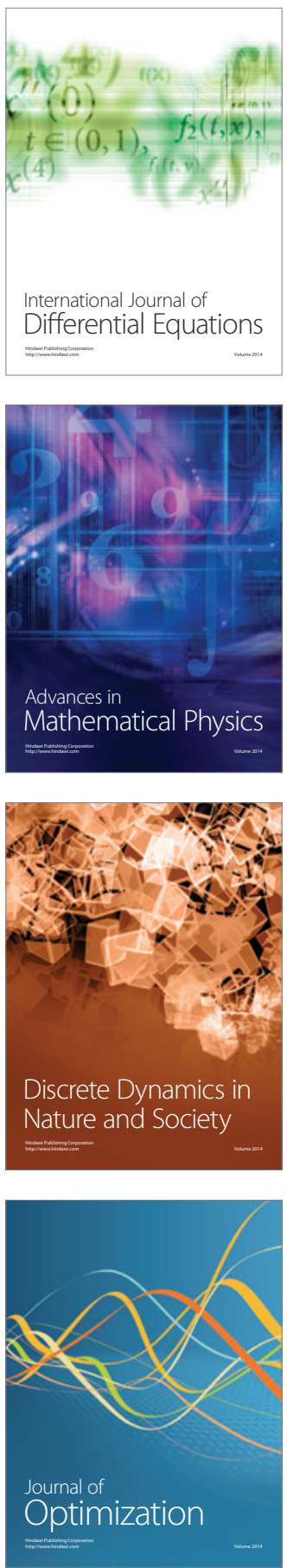